\title{
Development and Validation of an New High Performance Liquid Chromatography Method for Determination of Apixaban Isomers
}

\author{
Amit Gosar, Rajendra Phadke ", Deepesh Patil, Kushal Gupta, Prasad Thakur \\ Analytical Research \& Development Department, Indoco Remedies Limited, Navi Mumbai, India \\ Email address: \\ rajendraphadke75@gmail.com (R. Phadke) \\ ${ }^{*}$ Corresponding author \\ To cite this article: \\ Amit Gosar, Rajendra Phadke, Deepesh Patil, Kushal Gupta, Prasad Thakur. Development and Validation of an New High Performance \\ Liquid Chromatography Method for Determination of Apixaban Isomers. Chemical and Biomolecular Engineering. \\ Vol. 5, No. 1, 2020, pp. 1-7. doi: 10.11648/j.cbe.20200501.11
}

Received: October 14, 2019; Accepted: October 31, 2019; Published: January 4, 2020

\begin{abstract}
The purpose of this research study is to develop a novel, simple, precise, accurate and economical method for determination of Apixaban API isomers. Apixaban API has three isomer as ortho, meta and para. In reverse phase and normal phase chromatography it was very difficult to separate these isomer; hence new chiral technique was adopted. This chromatographic method was developed on chiralpak IA column $(250 \times 4.6 \times 5 \mu \mathrm{m})$ with isocritic technique. The detection of isomeric impurities were observed at wavelength $290 \mathrm{~nm}$. This analytical method was validated as per ICH guideline and regression analysis showed $\mathrm{R}$ value (correlation coefficient) $>0.999$ for Apixaban API and its isomeric impurities. A solution of Apixaban in dichloromethane was found stable up to $48 \mathrm{hrs}$. The degradation study was done within the given guidelines prescribed by ICH. The method is validated for Linearity, Accuracy and Precision.
\end{abstract}

Keywords: Apixaban, NP-HPLC, Isocritic, Validation, Chiralpak IA Column

\section{Introduction}

Apixaban is chemically known as 1-(4-methoxyphenyl)-7oxo-6-[4-(2-oxopiperidin-1-yl) phenyl]-4, 5, 6, 7-tetrahydro1H-pyrazolo [3, 4-c] pyridine-3-carboxamide is a Anti coagulant drug, a direct inhibitor of factor $\mathrm{X}$, also used in the prevention of venous thrombo embolism. Apixaban is recommended by the National Institute for Health and Clinical Excellence for the prevention of stroke and systemic embolism in people with non-valvular atrial fibrillation and at least one of the following risk factors: prior stroke or transient ischemic attack, age 75 years or older, diabetes mellitus, or symptomatic heart failure [1].

\section{Objectives of the Study}

The literature survey reveals that few analytical methods have been reported for Apixaban and its related compounds including spectroscopic methods, high performance liquid chromatography (HPLC) methods [7-11]. Our objective is to develop and validate new normal phase HPLC method for determination of Apixaban and its isomer. The proposed NPHPLC method utilizes economical solvent system having advantage like better retention time, peak sharp and symmetric nature. In reverse phase Chromatographic these impurities are not well separated from the product hence new chiral technique was developed. This method was validated according to ICH Guidelines.

\section{Instrumentation}

Waters, Alliance 2695 series HPLC system comprising a Quaternary pump, an auto-sampler, a thermostat column compartment, a solvent cabinet with gasser along with photo diode array (PDA) 2998 and ultraviolet (UV) 2487 detectors were used for separation and detection. Data acquisition and calculations were carried out using Waters Empower3 software (Milford). Sartorius (Germany) analytical balance was used for weighing material. 


\section{Materials and Reagent}

Apixaban sample, working standard and its related substances working standard were received from Analytical
Research and Development department of Indoco Research Centre (Navi Mumbai). HPLC grade n- Hexane, Toluene, Methanol, Ethanol and Dichloromethane were purchased from Merck (India).

Table 1. Chemical name of Apixaban and its isomers.

Sr No. Component Name

\subsection{Chromatographic Condition and Measurement Procedure}

\subsubsection{Mobile Phase}

Mix 65 volume of n-Hexane, 15 volume of Toluene, 10 volume of Methanol and 10 volume of Ethanol and degas by sonication for 2 mins.

\subsubsection{Diluent}

\section{Methanol}

\subsubsection{Preparation of Blank}

Transfer $5 \mathrm{ml}$ Dichloromethane into $10 \mathrm{ml}$ volumetric flask and make up with diluent.

Table 2. Chromatographic Conditions.

\begin{tabular}{ll}
\hline Column & Chiralapk IA (250mm x 4.6 mm, 5 $\mathbf{m m})$ \\
\hline Column Temperature & $25^{\circ} \mathrm{C} \pm 2{ }^{\circ} \mathrm{C}$ \\
Flow Rate & $1.0 \mathrm{~mL} / \mathrm{min}$ \\
Injection Volume & $30 \mu \mathrm{L}$ \\
Detector Wavelength & $290 \mathrm{~nm}$ \\
Run Time & 60 minutes \\
Retention Time & Apixaban (APX) about 32 minutes, \\
Needle wash & Dichloromethane \\
\hline
\end{tabular}

\subsection{Preparation of Solutions}

\subsubsection{System Suitability Solution}

Transfer about $5 \mathrm{mg}$ of Apixaban working standard, Apixaban ortho impurity and Apixaban meta impurity into 10 $\mathrm{mL}$ volumetric flask, add $5 \mathrm{ml}$ dichloromethane and dissolve in and make upto the mark with diluent.

\subsubsection{Reference Solution (a)}

Transfer about $60 \mathrm{mg}$ of Apixaban working standard into $10 \mathrm{~mL}$ volumetric flask, dissolve in about $5 \mathrm{~mL}$ of dichloromethane and make upto the mark with diluent.
Transfer $1 \mathrm{~mL}$ of above solution to $100 \mathrm{~mL}$ volumetric flask and make upto the mark with diluent. Further transfer 1 $\mathrm{mL}$ of above solution into $10 \mathrm{~mL}$ volumetric flask and make upto the mark with diluent.

\subsubsection{Test Solution}

Transfer about $60 \mathrm{mg}$ of Apixaban sample into $10 \mathrm{~mL}$ volumetric flask, dissolve in about $5 \mathrm{~mL}$ of dichloromethane and make upto the mark with diluent.

Table 3. Injection sequence.

\begin{tabular}{lll}
\hline SI\# & Description & No. of Injections \\
\hline 1 & Blank & 1 \\
2 & System suitability solution & 1 \\
3 & Blank & 1 \\
4 & Reference solution (a) & 5 \\
5 & Test solution & 2 \\
\hline
\end{tabular}

\subsubsection{Procedure}

Equilibrate the HPLC system with the initial composition until a steady baseline is obtained. Inject Blank, System suitability solution and reference solution (a). Ensure that all the system suitability parameters meet the requirements. Inject test solution as per injection sequence and record the chromatograms. Make blank correction if necessary.

Table 4. Peak name with Retention Time and Relative Response Factor.

\begin{tabular}{llll}
\hline Sr. No & Peak Name & $\begin{array}{l}\text { Relative } \\
\text { Retention Time }\end{array}$ & $\begin{array}{l}\text { Relative Retention } \\
\text { Factor }\end{array}$ \\
\hline 1 & Apixaban & 1.00 & 1.00 \\
2 & Ortho impurity & 0.58 & 0.55 \\
3 & Meta impurity & 0.90 & 0.80 \\
\hline
\end{tabular}

\subsection{System Suitability [3]}

\subsubsection{Acceptance Criteria}

Resolution: The resolution between the peaks due to meta impurity and Apixaban in the chromatograms obtained with 
system suitability solution should not be less than 1.5.

\%RSD: The percent relative standard deviation of three replicates for the peak due to Apixaban in the chromatograms obtained with reference solution (a) should not be more than 5.0.

\subsubsection{Calculation}

Calculate impurity content by formula given below:

$$
\% \text { Ortho/Meta impurity }=\frac{\mathrm{AI} \times \mathrm{WS} \times 1 \times 1 \times 1}{\mathrm{AR} \times \mathrm{WT} \times 100 \times 10 \times \mathrm{RRF}} \times \mathrm{P}
$$

Where,

$\mathrm{AI}=$ Average peak area for respective impurity in test solution.

$\mathrm{AR}=$ Average peak area of Apixaban in reference solution (a).

WS $=$ Weight in mg of Apixaban working standard taken for reference solution (a) preparation.

$\mathrm{WT}=$ Weight in mg of Apixaban sample taken for test solution preparation.

$\mathrm{P}=$ Potency of Apixaban working standard (\%).

\subsubsection{Analytical Method Validation}

The developed method is subjected to analytical method validation, which is conducted according to International Council for harmonization (ICH) guidelines. The parameters with which analytical method is validated are specificity, limit of detection and limit of quantification, linearity, accuracy, precision [4-6].

\subsection{Specificity}

Specificity is capability of the method to measure the analyte response in presence of impurities. The typical chromatograms of blank Solution, System suitability solution, Reference Solution (a), and Impurities Spike Solution are given from figure 1 to figure 4 respectively. The results indicate that all impurities are well separated under the current chromatographic conditions. There was no interference of peak from blank solution and samples solution within retention time of impurities obtained. Peak purity for Apixaban and its impurities were passing. For retention time of each impurity and its peak purity refer table no.5

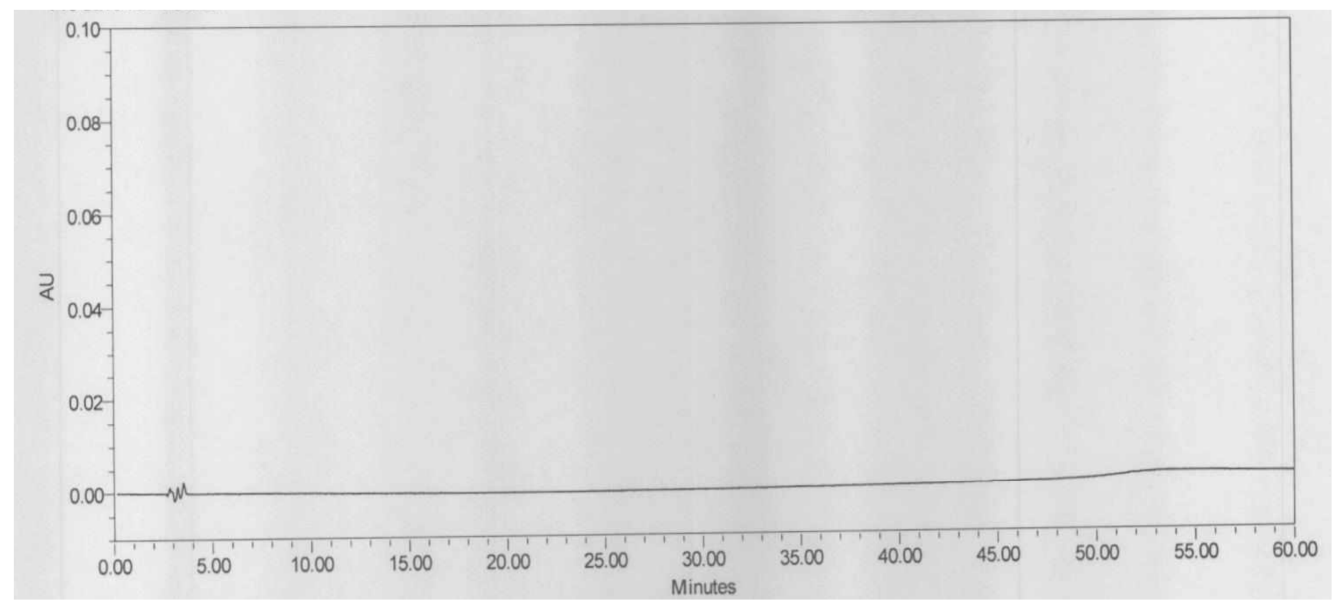

Figure 1. Blank

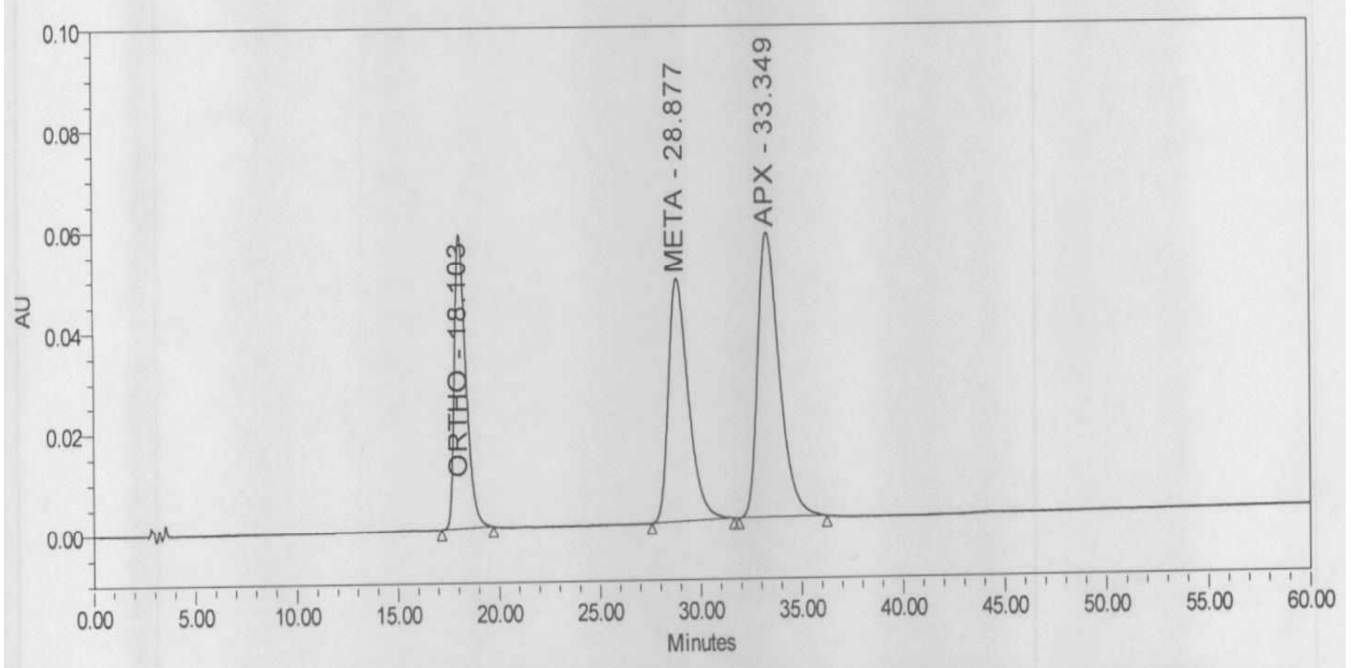

Figure 2. System suitability solution. 


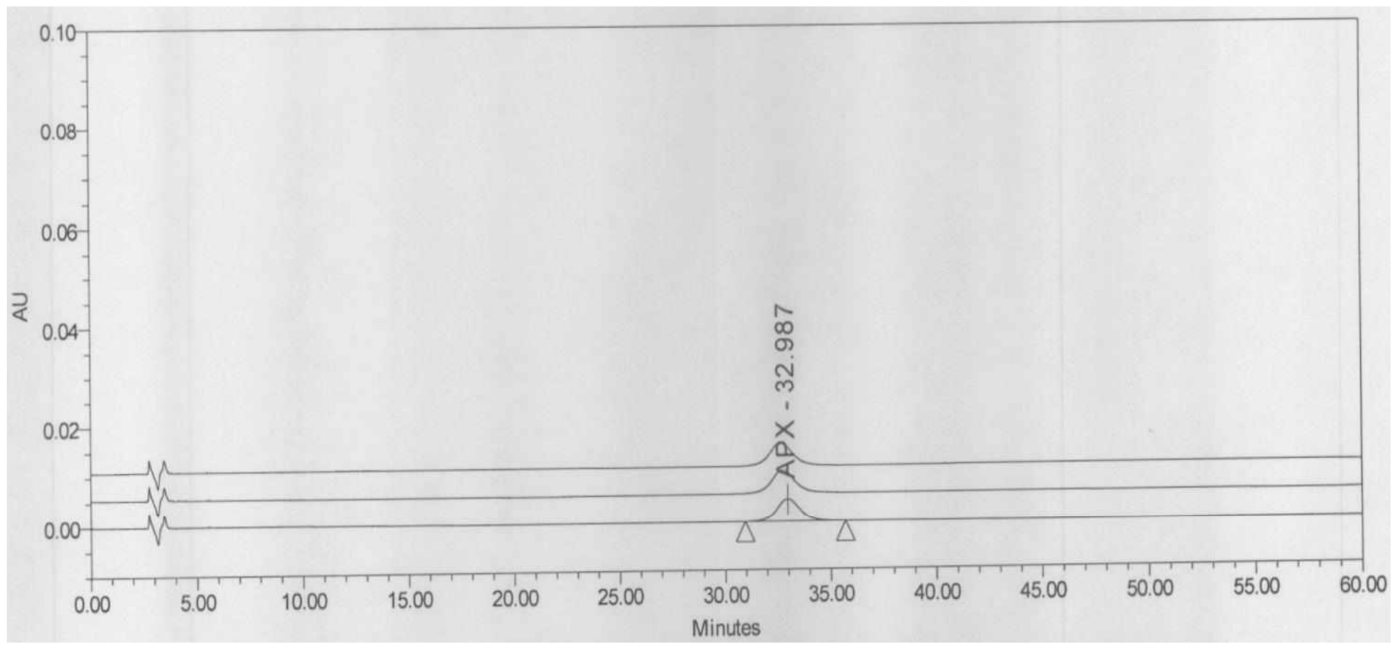

Figure 3. Reference Solution (a)

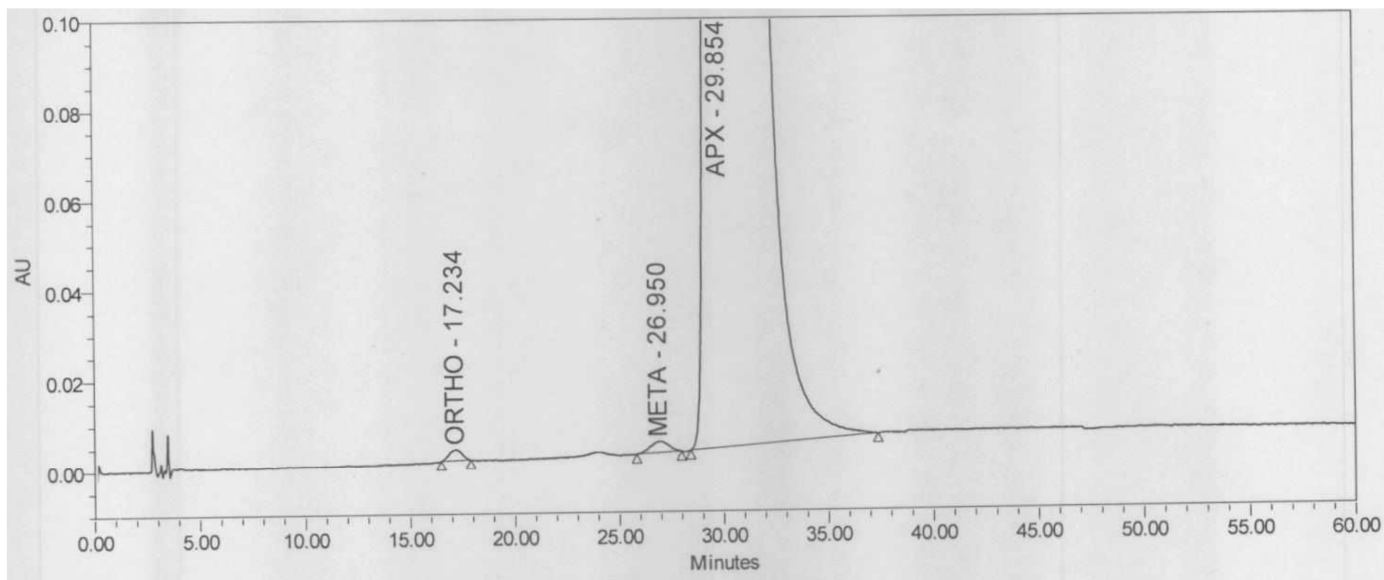

Figure 4. Impurities spiked in sample.

Table 5. Peak purity and RT Ratio for Apixaban and its impurities.

\begin{tabular}{llllll}
\hline Sr. no & Peak name & RT & RT Ratio & Purity Angle & Purity Threshold \\
\hline 1 & Apixaban & 32.0 & 1.00 & 0.22 & 1.18 \\
2 & Ortho impurity & 18.56 & 0.58 & 0.19 & 1.17 \\
3 & Meta impurity & 28.8 & 0.90 & 0.22 & 1.16 \\
\hline
\end{tabular}

\subsection{Limit of Detection and Limit of Quantification}

Series of standard solution of Apixaban and its impurities were prepared and injected in concentration ranging from $50 \%$ to $500 \%$. Limit of detection (LOD) and Limit of quantification (LOQ) was calculated based on residual standard deviation of regression line and slope. Both calculated LOD and LOQ were well within limit. The LOQ is below $0.057 \%$ for impurities and Apixaban API. For details of LOQ and LOD refer Table.

Table 6. $L O D$ and $L O Q$.

\begin{tabular}{llll}
\hline Sr No. & Name of Impurity & LOD (\%) & LOQ (\%) \\
\hline 1 & Apixaban & 0.019 & 0.057 \\
2 & Ortho impurity & 0.018 & 0.055 \\
3 & Meta impurity & 0.011 & 0.032 \\
\hline
\end{tabular}

\subsection{Linearity}

Series of linearity solution of Apixaban and its impurities were prepared from $50 \%$ to $500 \%$ of test concentration. Linearity curves were drawn by plotting the peak areas of Apixaban and its impurities against its corresponding concentration of linearity solution. Observed regression coefficient was greater than 0.998 and \% y-intercept was less than $5.0 \%$

Table 7. Linearity table and its $R^{2}$ values Apixaban.

\begin{tabular}{ll}
\hline Slope & 2265608.63 \\
Intercept & 19532.23 \\
Co-relation Coefficient $\left(\mathrm{R}^{2}\right)$ & 0.9993 \\
$\%$ Y-Intercept & 2.77 \\
\hline
\end{tabular}


Apixaban

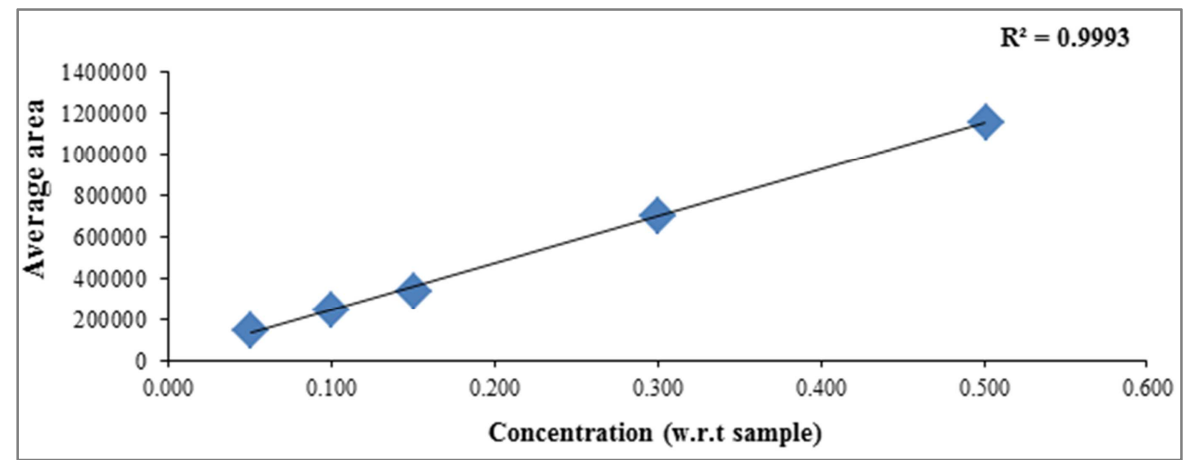

Figure 5. Linearity graph of Apixaban.

Table 8. Linearity table and its $R^{2}$ values of Meta Impurity.

\begin{tabular}{ll}
\hline Slope & 1757553.28 \\
Intercept & -17795.47 \\
Co-relation Coefficient (R2) & 0.9998 \\
$\%$ Y-Intercept & -3.37 \\
\hline
\end{tabular}

\section{Meta Impurity}

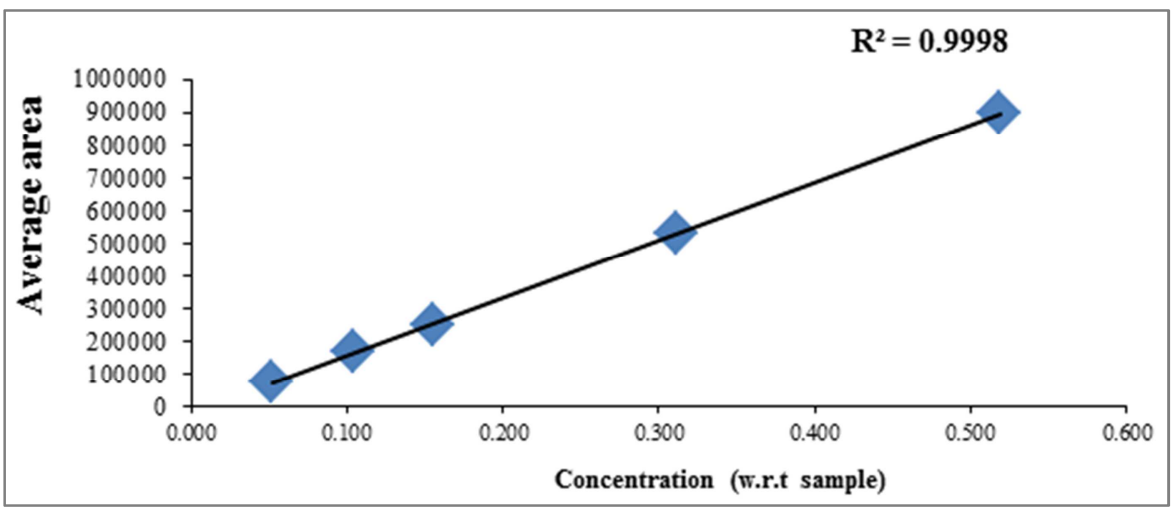

Figure 6. Linearity graph of Meta Impurity.

Table 9. Linearity table and its $R^{2}$ values of Ortho Impurity.

\begin{tabular}{ll}
\hline Slope & 1239296.68 \\
Intercept & -7260.83 \\
Co-relation Coefficient $\left(\mathrm{R}^{2}\right)$ & 0.9994 \\
$\%$ Y-Intercept & -4.02 \\
\hline
\end{tabular}

Ortho Impurity

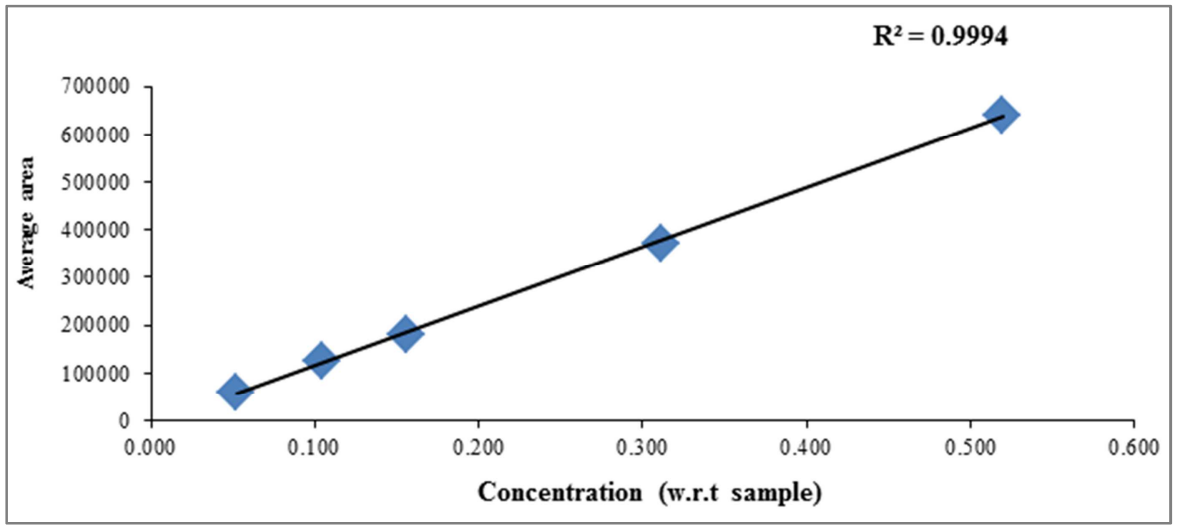

Figure 7. Linearity graph of Ortho Impurity. 


\subsection{Accuracy}

Accuracy of method is calculated and established by carrying out recovery studies of impurities. The test solution was spiked with impurities solution at specific limit level concentration $100 \%, 150 \%, 300 \%$. Each spiked test solution was analyzed for recovery study of impurities. Recovery established is between $100 \%$ to $105 \%$.

Table 10. Recovery of impurities at $100 \%$.

\begin{tabular}{llllll}
\hline Sr. No. & Imp Name & Area of $\mathbf{0 . 1 0} \%$ Level & Theoretical imp Added \% & \% of IMP Observed & \% of Recovery \\
\hline 1 & Ortho Impurity & 103945 & 0.09 & 0.09 & 103.15 \\
2 & Meta Impurity & 147724 & 0.09 & 0.09 & 100.78 \\
\hline
\end{tabular}

Table 11. Recovery of impurities at $150 \%$.

\begin{tabular}{llllll}
\hline Sr. No. & IMP Name & Area of $\mathbf{0 . 1 5 \%}$ Level & Theoretical imp Added \% & \% of IMP Observed & \%of Recovery \\
\hline 1 & Ortho Impurity & 160337 & 0.13 & 0.14 & 104.56 \\
2 & Meta Impurity & 226362 & 0.13 & 0.14 & 101.49 \\
\hline
\end{tabular}

Table 12. Recovery of impurities at $300 \%$.

\begin{tabular}{llllll}
\hline Sr. No. & IMP Name & Area of $\mathbf{0 . 3 0} \%$ Level & Theoretical imp Added \% & \% of IMP Observed & \%of Recovery \\
\hline 1 & Ortho Impurity & 319004 & 0.27 & 0.28 & 104.88 \\
2 & Meta Impurity & 444756 & 0.27 & 0.27 & 100.52 \\
\hline
\end{tabular}

\subsection{Precision}

System precision was carried out by analyzing six injections of reference solution (a) of Apixaban API at limit level concentration. Relative standard deviation for peak area of Apixaban was calculated and found to be $0.33 \%$.

\subsection{Robustness}

Robustness was studied by making small but deliberate changes in optimized method conditions and evaluating the effect on resolution between ortho impurity, meta impurity and Apixaban. The mobile phase flow rate was changed by \pm 0.1 units from 1.0 to 0.9 and $1.1 \mathrm{ml} / \mathrm{min}$.

\subsection{Solution Stability}

The solution stability of Apixaban and its impurities in related substances method was evaluated by leaving a spiked solution in tightly capped volumetric flask at room temperature for $48 \mathrm{hrs}$ and analysis of all its impurities content was done at interval of $12 \mathrm{hrs}$.

\section{Result and Discussion}

Since most of the impurities are non-polar in nature, various chiral columns were screened. The parameters like tailing factor and theoretical plates were recorded during the study. From obtained data Chiralpak IA $(250 \times 4.6 \times 5 \mu \mathrm{m})$ was found suitable for analysis.

All impurities were prepared at $100 \mathrm{ppm}$ and their UVvisible spectra was acquired. The Apixaban and all its impurities has good and satisfactory response at $290 \mathrm{~nm}$. Hence detection at $290 \mathrm{~nm}$ was selected for methoddevelopment.

Retention time were confirmed by injecting working standards of Apixaban, Meta and ortho impurities. For System Suitability, the resolution between Apixaban and Meta imp should be not less then 1.5. Relative Standard Deviation for five replicate injections peak due to Apixaban should not be more then $5 \%$.

\section{Conclusion}

The analytical method validation for Apixaban by normal Phase HPLC was carried out by performing the parameters such as specificity, limit of detection and limit of quantification, linearity, accuracy, precision, robustness and solution stability. All the data has been compiled and found to be satisfactory. Hence, method developed for normal Phase HPLC can be suitably used for analysis of Apixaban isomer in pharmaceutic industries.

\section{Acknowledgements}

The author wishes to thank to management of Indoco Remedies Limited for supporting this work by providing the samples of Apixaban API and its impurities working standard required for this research.

\section{Authors' Contributions}

This work was carried out in collaboration between all authors. All available literature search and regulatory requirement author AG and RP designed the study. Authors DP and KG wrote the protocol and authors PT carried out the experiment along with KG. All author involved in statistical analysis and writing the first draft of manuscript and subsequent revision. All the authors read and approved the final manuscript. 


\section{References}

[1] Indian Pharmacopoeia, volume III, Ministry of Health and Family Welfare Government of India. Published by Indian Pharmacopoeia Commission, Ghaziabad, 2014.

[2] The British Pharmacopoeia, the British Pharmacopoeia Commission, London. 2012; Vol. II.

[3] The United state Pharmacopoeia, the United state Pharmacopoeia Commission, America. 2012.

[4] ICH Harmonized Tripartite Guideline, Validation of analytical procedure text and methodology Q2 [R1]. In International Conference on Harmonization of technical requirements for registration of pharmaceuticals for human use, 2005.

[5] AM K, Vidhate MB, Ingale MR. Analytical method development and validation for estimation of apixaban by RPHPLC. Indian Drugs, 2017; Vol. 54 (4); 76-79.

[6] Sun YY, Zhao YL, Duan MM, Wang DK, Yu ZG. Determination of related substances in apixaban by HPLC. Journal of Shenyang Pharmaceutical University. 2017 (1): 7.

[7] Mahendra B, Sundari KH, Vimalakkannan T. Method developed for the determination of apixaban by using UV spectrophotometric. International Journal of Research In
Pharmaceutical Chemistry and Analysis. 2019 Jul 29; 1 (3): $83-7$.

[8] El-Bagary RI, Elkady EF, Farid NA, Youssef NF. Validated spectrofluorimetric methods for the determination of apixaban and tirofiban hydrochloride in pharmaceutical formulations. Spectrochimica Acta Part A: Molecular and Biomolecular Spectroscopy. 2017 Mar 5; 174: 326-30.

[9] Bhavesh R. Sharma Chainesh N. Shah, A Recent Research Review- Development and Validation with liquid chromatography, J Pharm Sci Bioscientific Res. 2016. 6 (5): 723-727.

[10] Rajan ST, Eswaraiah S, Venkatesh M, inventors; Msn Laboratories Limited, assignee. Intermediate and polymorphs of 1-(4-methoxypheny1)-7-oxo-6-[4-(2-oxopiperidin-1-yl) phenyl]-4, 5, 6, 7-tetra hydro-1H-pyrazolo [3, 4-c] pyridine-3carboxamide and process thereof. United States patent US 9,718,779. 2017 Aug 1.

[11] Vemula Archana Reddy, Method development and validation of apixaban in bulk and tablet dosage form by RP-HPLC. Journal of Pharmacreations Vol-5 (1) 2018 [01-24]

[12] D. Shravan Kumar, Dr. M. Ajitha, Mahesh Rajendra Awate, K. S. L. Harika, stability indicating assay method development and validation of apixaban tablets by RP-HPLC. J Sci Res Pharm, 2017; 6 (Suppl 1): 71-75. 